\title{
Maxwell's Equal Area Law and the Hawking-Page Phase Transition
}

\author{
Euro Spallucci and Anais Smailagic \\ Dipartimento di Fisica Teorica, Università di Trieste and INFN, 34151 Sezione di Trieste, Italy \\ Correspondence should be addressed to Euro Spallucci; euro@ts.infn.it
}

Received 24 June 2013; Revised 12 September 2013; Accepted 8 October 2013

Academic Editor: Jose Antonio De Freitas Pacheco

Copyright (C) 2013 E. Spallucci and A. Smailagic. This is an open access article distributed under the Creative Commons Attribution License, which permits unrestricted use, distribution, and reproduction in any medium, provided the original work is properly cited.

\begin{abstract}
We study the phases of a Schwarzschild black hole in the Anti-deSitter background geometry. Exploiting fluid/gravity duality, we construct the Maxwell equal area isotherm $T=T^{*}$ in the temperature-entropy plane, in order to eliminate negative heat capacity BHs. The construction we present here is reminiscent of the isobar cut in the pressure-volume plane which eliminates unphysical part of the Van der Walls curves below the critical temperature. Our construction also modifies the Hawking-Page phase transition. Stable BHs are formed at the temperature $T>T^{*}$, while pure radiation persists for $T<T^{*} . T^{*}$ turns out to be below the standard Hawking-Page temperature and there are no unstable BHs as in the usual scenario. Also, we show that, in order to reproduce the correct $\mathrm{BH}$ entropy $S=A / 4$, one has to write a black hole equation of state, that is, $P=P(V)$, in terms of the geometrical volume $V=4 \pi r_{H}^{3} / 3$.
\end{abstract}

\section{Introduction}

Black holes (BHs) are among the most intriguing solutions of Einstein equations. Their geometric description is fully provided by the theory of general relativity and is discussed in many excellent textbooks. However, this is only half of the story.

Since the original works by Bekenstein and Hawking, some new aspects of the $\mathrm{BH}$ behavior emerged once quantum field theory is coupled to a BH background geometry. Even if this is only a "semiclassical" quantum gravity formulation, the outcome has profoundly changed the prospective of the $\mathrm{BH}$ behavior.

A stellar mass, classical, $\mathrm{BH}$ is characterized by the unique feature of being a perfect absorber with a vanishing luminosity. From a thermodynamical point of view, a classical $\mathrm{BH}$ is a zero temperature black body.

However, nuclear size BHs, interacting with quantized matter, are almost perfect black bodies as they emit black body radiation at a characteristic nonvanishing temperature! Moreover, BHs are assigned a thermodynamical property identified with entropy. Thus, there are two complementary descriptions of $\mathrm{BH}$ physics: one in terms of pure space-time geometry and the other in terms of thermodynamics. The two descriptions are related to each other through the so-called "first law" of BH (thermo)dynamics as follows:

$$
d M=T d S+\phi_{H} d Q+\Omega_{H} d J,
$$

where $M=$ total mass energy, $T=$ Hawking temperature, $S=$ entropy, $\phi_{H}=$ the Coulomb potential on the horizon, $Q=$ electric charge, $\Omega_{H}=$ angular velocity of the horizon, and $J=$ angular momentum.

The first law (1) is the basis of the thermodynamical description of the $\mathrm{BH}$ as a "fluid" where $d M$ is the variation of total energy split into variation of "internal," "electrostatic," and "rotational" pieces, which are then given a thermodynamical meaning. By comparing (1) to the first law as it appears in fluid thermodynamics, one cannot but notice the absence of a fundamental pair of canonical thermodynamical variables, that is, the volume and the pressure of the system. While the volume in question can be naturally identified with the volume excluded by the event horizon [1], it is a bit ambiguous what geometrical quantity is to be identified with pressure [2-5]. However, for BHs in Anti-deSitter background, there is a natural candidate, that is, the cosmological constant [6-12]. In fact, AdS/dS spaces can be sourced by 
an energy-momentum tensor satisfying the vacuum state equation

$$
P=-\rho=-\frac{\Lambda}{8 \pi G_{N}} .
$$

With this identification, it is possible to write the extended first law in its simplest form as

$$
d M=T d S+V d P .
$$

Equation (3) can be rewritten as

$$
d(M-P V)=T d S-P d V,
$$

which allows the identification of the proper thermodynamical potential for the $\mathrm{BH}$ :

$$
\begin{gathered}
U=M-P V, \\
M \equiv H=U+P V, \\
F=U-T S, \\
G=H-T S,
\end{gathered}
$$

where $U$ is the internal energy, $H$ is the enthalpy, $F$ is the Helmholtz free energy, and $G$ is the Gibbs free energy.

Equation (3) envisages the $\mathrm{BH}$ as a "gas" enclosed in its geometrical volume, $V$, and subject to an external pressure $P$ given in (2). The question naturally arises: what is the equation of state for the gas?

The answer is as follows: take the expression of the Hawking temperature in terms of the geometrical quantities, that is, radius of the event horizon and $\Lambda$, replace $\lambda$ with $P$, and solve $P=P(T, V)$. The $\mathrm{BH}$ fluid equation makes it possible to investigate the existence of different "phases" of the system and the kind of changes of state [13-16].

Recently, this approach has led to the identification, for charged AdS $\mathrm{BH}$, of the pressure equation with the one of a Van der Waals gas [2-4]. Once this identification is achieved, one can pursue the standard Van der Waals analysis of phase transitions, calculate the critical exponents, and apply the Maxwell equal area construction in the $(P, V)$ plane. At this point, it is worth mentioning that the Maxwell equal area construction can be equally applied in the $(T, S)$ plane at constant pressure. This has been done in [17] with interesting results: (i) the equal area law can be analytically solved; (ii) the unphysical negative specific heat region is cutoff; (iii) a new $\mathrm{BH}$ phase structure emerges; (iv) the role of the Van der Waals unshrinkable molecular volume is taken by the extremal $\mathrm{BH}$ configuration, thus justifying its stability.

The Van der Waals description of charged BHs assumes a particular interest in the framework of AdS/CFT duality which conjectures a relation between QCD and real gas phase transitions.

For neutral, nonrotating, BHs in AdS background, the liquid-gas coexistence is absent in the sense that below certain temperature there are no more BHs. However, in this case, one speaks of a different type of phase transition between a pure background radiation and stable/unstable BHs [18].
In this paper, we will apply the Maxwell equal area construction in the $(T, S)$ plane to the neutral $\mathrm{BH}$ in the AdS background and study how the Hawking-Page phase transition is affected. Although the model does not belong to the same universality class of charged AdS BHs, it still has all the thermodynamical potentials necessary for the Maxwell equal area law construction.

\section{Neutral AdS Black Hole and the Area Law}

2.1. Review of the Hawking-Page Phase Transition. We start from the Schwarzschild Anti-deSitter (SAdS) metric. The line element is given by $\left(G_{N}=1\right.$ units $)$

$$
\begin{aligned}
d s^{2}= & -\left(1-\frac{2 M}{r}+\frac{r^{2}}{l^{2}}\right) d t^{2}+\left(1-\frac{2 M}{r}+\frac{r^{2}}{l^{2}}\right)^{-1} d r^{2} \\
& +r^{2} d \Omega_{(2)}^{2},
\end{aligned}
$$

where $M$ is the mass and the AdS curvature radius $l$ is related to the cosmological constant as $\Lambda=-3 / l^{2}$. We briefly review the properties of the solution (6) which are relevant for our discussion.

The horizon is determined by the zero of the $g_{r r}^{-1}$ metric component as follows:

$$
M=\frac{r_{+}}{2}\left(1+\frac{r_{+}^{2}}{l^{2}}\right) .
$$

In the case of Schwarzschild AdS equation (7) shows that for any positive value of $M$ there exists only one horizon. As a consequence, this kind of $\mathrm{BH}$ does not admit any extremal configuration where $M$ has a minimum. As a function of $r_{+}, M$ is a monotonically increasing function linearly vanishing as $r_{+} \rightarrow 0$.

The corresponding Hawking temperature $T_{H}$ is given by (see Figure 1)

$$
k_{B} T=\frac{1}{4 \pi r_{+}}\left(1+\frac{3 r_{+}^{2}}{l^{2}}\right) .
$$

Equation (8) can be inverted by expressing $r_{+}$as a function of $T$ as the free variable. Written in this form, (8) defines the existence of multiple $\mathrm{BH}$ at the same temperature as follows:

$$
\frac{3}{l^{2}} r_{+}^{2}-4 \pi k_{B} T r_{+}+1=0
$$

This is a quadratic algebraic equation admitting real solutions for

$$
T \geq T_{\min } \equiv \frac{\sqrt{3}}{2 \pi l} .
$$

For $T<T_{\min }$, there are no BHs and we are in a pure radiation phase. The background heat bath is too cold to admit nucleation of BHs.

For $T=T_{\min }$, a single $\mathrm{BH}$ is formed with a radius

$$
\widehat{r}=\frac{l}{\sqrt{3}} \text {. }
$$




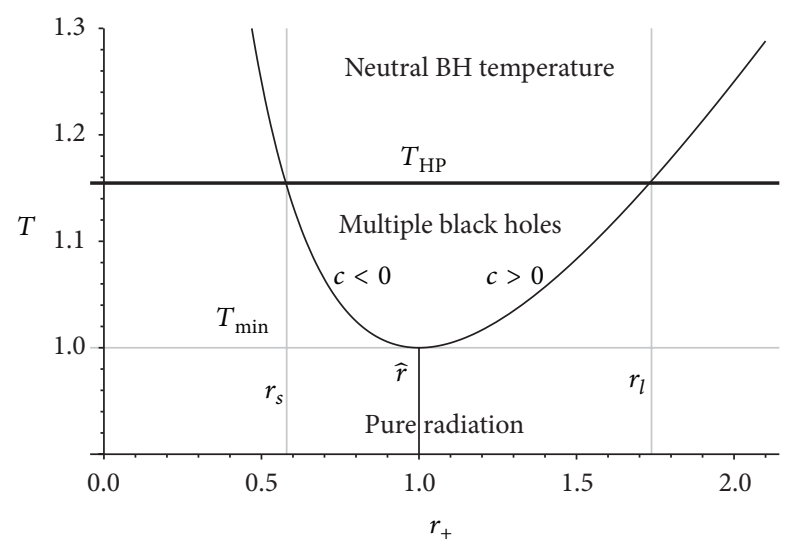

FIgUre 1: Plot of the Hawking temperature showing Hawking-Page phases. For $T<T_{\min }$, there are no BHs but a pure radiation phase. For $T>T_{\min }$, there exists a pair of BHs with radii $r_{l}, r_{s}$ given in (12). The smaller $\mathrm{BH}$ is (thermodynamically) unstable, while the larger is either locally stable, $T<T_{\mathrm{HP}}$, or globally stable for $T>T_{\mathrm{HP}}$.

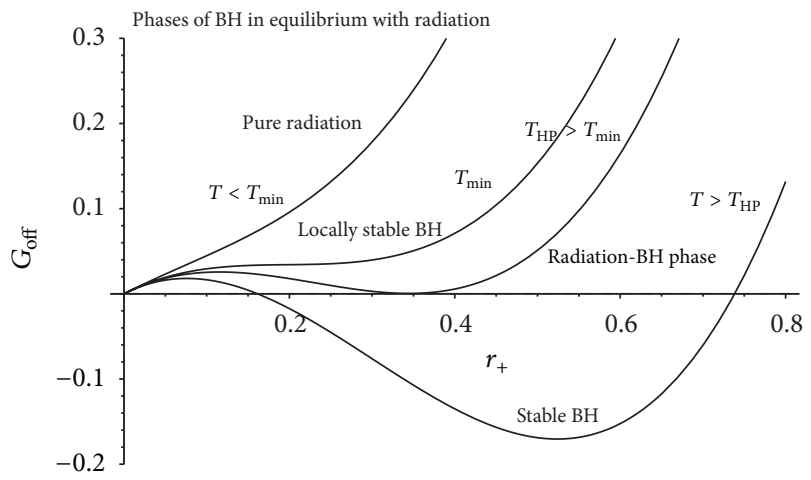

Figure 2: Plot of the Gibbs free energy for different temperatures.

For $T>T_{\min }$, a pair of BHs (large/small) exists with radii given by

$$
r_{l, s}=\frac{T}{2 \pi T_{\min }^{2}}\left(1 \pm \sqrt{1-\frac{T_{\min }^{2}}{T^{2}}}\right)
$$

where $r_{s}<\widehat{r}$ and $r_{l}>\widehat{r}$. In order to investigate the stability of small and large BHs, we look at the Gibbs free energy (see Figure 2)

$$
G \equiv M-T S=\frac{r_{+}}{2}\left(1+\frac{r_{+}^{2}}{l^{2}}\right)-\pi k_{B} T r_{+}^{2} .
$$

BHs in thermal equilibrium with the background correspond to stationary points of $G$ for $T>T_{\min }$. At a lower temperature, the single, global, minimum of $G$ is the origin, that is, $r_{+}=0$, and the system is in a pure radiation phase.

For $T=T_{\min }, G$ exhibits an inflexion point for $r_{+}=$ $1 / 2 \pi T_{\min }=l / \sqrt{3}$. From this temperature on, we recover two $\mathrm{BHs}$ with radii given by (12). The smallest corresponds to a local maximum of $G$, while the larger one is locally stable being a local minimum. With increasing $T$, the local minimum of $G$ lowers until reaching zero for

$$
T=\frac{1}{\pi l} \equiv T_{\mathrm{HP}}
$$

where $T_{\mathrm{HP}}$ is known as the Hawking-Page critical temperature and where the Gibbs energy of a $\mathrm{BH}$ of radius $r_{+}=l$ is degenerate with the Gibbs free energy of a pure radiation at the same temperature. In this case, a BH with radius $r_{+}=l$ is degenerate with the background radiation. Finally, for $T>$ $T_{\mathrm{HP}}$, large BHs are globally stable, absolute minima of $G$.

This is a brief summary of the radiation into $\mathrm{BH}$ transition as originally introduced by Hawking and Page.

The question of stability of the BHs is also related to the sign of the constant specific heat [10] as follows:

$$
C_{P} \equiv\left(\frac{\partial M}{\partial T}\right)_{P}=-2 \pi r_{+}^{2}\left(1+\frac{3 r_{+}^{2}}{l^{2}}\right)\left(1-\frac{3 r_{+}^{2}}{l^{2}}\right)^{-1},
$$

where $C_{P}<0$ describes unstable $\mathrm{BH}$ corresponding to local maxima of $G$, which cannot be in thermal equilibrium with the surrounding heat bath.

In this paper, we will discuss how to remove negative specific heat BHs by applying the Maxwell equal area construction and see how it affects the Hawking-Page phase structure.

2.2. The Gas Equation. Recently, a number of papers have dealt with the analogy between BHs in AdS and Van der Walls "real" fluids. From this vantage point, particular attention has been given to charged and/or rotating BHs. This approach is made possible if one assigns the cosmological constant the role of pressure in the dual VdW picture. The advantage of the dual picture is that one can exploit well-known properties and computational techniques from the WdV fluid dynamics, to gain a deeper understanding of $\mathrm{BH}$ thermodynamics, such as critical behavior and phase transitions. Furthermore, it is known that phase transitions in real fluids match the experimental results only if one applies an isothermal cut, the Maxwell equal area law, in the $(P, V)$ plane.

By exploiting this kind of duality, we have shown in a recent paper that the equal area construction can be implemented in the gravitational sector as well [17]. Working in $(T, S)$ plane, where $T$ and $S$ are the Hawking temperature and the $\mathrm{BH}$ entropy, has given a significant advantage in the sense that the area law can be solved analytically and the negative heat capacity region can be eliminated.

In this paper, we will extend the same approach to the Schwarzschild AdS case. While the liquid-gas transition in the $\mathrm{WdV}$ fluid is dual to a small-large $\mathrm{BH}$ change in the gravitational picture, the Hawking-Page transition, for neutral $\mathrm{BHs}$, has been interpreted by Witten as the gravitational dual of the QCD deconfinement transition $[19,20]$. Thus, the study of the Schwarzschild AdS BH properties can shed more light on the quark-gluon plasma properties presently subject to an intensive research interest [21]. 
In order to obtain the fluid picture of the SAdS BH, we rewrite (8) as

$$
P=\frac{k_{B} T}{2 r_{+}}-\frac{1}{8 \pi r_{+}^{2}},
$$

where the duality "dictionary" called for substitution:

$$
-\frac{\Lambda}{8 \pi} \longrightarrow P .
$$

The graph of $P$ for constant $T$ is given in Figure 3. One sees that $P$ vanishes at $r_{0}=1 / 4 \pi k_{B} T$ and reaches maximum $P_{M}=\pi k_{B}^{2} T^{2} / 2 G_{N}$ at $r_{M}=2 r_{0}$.

Although the graph is not of $\mathrm{VdW}$ form, the equal area law can be still defined between $r_{0}$ and $r_{g}$ which will be determined later. In order to make (16) look like the state equation of a fluid, we still need to associate a proper volume to the system.

In previous works $[22,23]$, the specific volume of the fluid was identified as $v=2 r_{H} G_{N}$. With respect to this choice, we cannot help but notice the following.

(i) The conjugate canonical variable to the cosmological constant (identified with the inward pressure) is the geometric volume $V=4 \pi r_{H}^{3} / 3$ of the $\mathrm{BH}$ since

$$
V \equiv \frac{\partial H}{\partial P}=\frac{4 \pi}{3} r_{+}^{3}
$$

where $H$ is given by (7).

(ii) Furthermore, it is widely accepted that the $\mathrm{BH}$ entropy is given by the area law $S=\pi r_{+}^{2}$. On the other hand, the entropy of a gas is logarithmic in the specific volume $v$.

Therefore, in order to satisfy both requirements, one has to express (16) in terms of the geometric volume as

$$
P=\frac{k_{B} T}{2}\left(\frac{4 \pi}{3 V}\right)^{1 / 3}-\frac{1}{8 \pi}\left(\frac{4 \pi}{3 V}\right)^{2 / 3},
$$

where $k_{B}$ is the Boltzman constant.

To compute the entropy, we start from the general formula for a gas:

$$
d S=C_{V} \frac{d T}{T}+\left(\frac{\partial P}{\partial T}\right)_{V} d V
$$

where $C_{V}$ is the specific heat at constant volume. Thus, (19) leads to

$$
\begin{gathered}
d S=\frac{k_{B}}{2}\left(\frac{4 \pi}{3}\right)^{1 / 3} \frac{d V}{V^{1 / 3}}, \\
S=k_{B}\left(\frac{A}{4}\right) .
\end{gathered}
$$

We find the $\mathrm{BH}$ area law for entropy. Therefore, the area law follows from the gas equation only in terms of the geometric volume of the $\mathrm{BH}$.

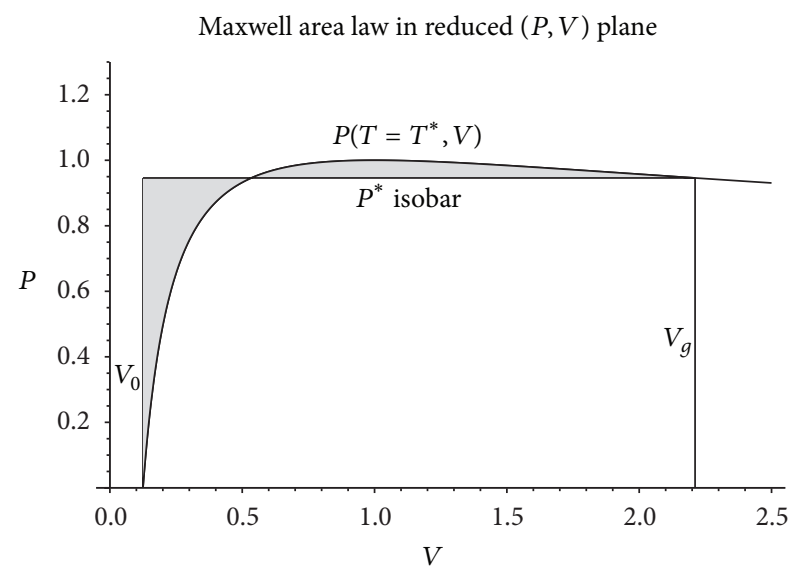

FIgURE 3: Plot of the (rescaled) pressure at fixed $T$, as a function of the geometric volume of the $\mathrm{BH}$. Equal area regions are colored in black.

\section{Equal Area Maxwell Construction}

The equal area law was introduced by Maxwell in order to explain the experimental behavior of real fluid. Normally, this construction is applied in the $(P, V)$ plane keeping the temperature constant. Theoretically, it can be derived from the variation of the Gibbs free energy $G$ :

$$
d G=-S d T+V d P .
$$

At constant $T$, one finds the equal area law:

$$
P^{*} \Delta V=\int_{V_{l}}^{V_{g}} P d V
$$

where $P=P^{*}$ is an isobar defining equal areas and $V_{l}, V_{g}$ are the volume of the liquid and gaseous phase. However, if one keeps the pressure constant in (22), the same construction can be done in the $(T, S)$ plane.

In this paper, we construct the Maxwell area law in the latter plane for two reasons:

(i) it is through the temperature graph that the HawkingPage phase transition has been originally studied [18], and we are interested in the modification of this phase structure;

(ii) we have shown in [17] that in this plane the equation for the equal area law can be explicitly solved.

We start from the temperature as a function of entropy given by

$$
k_{B} T=\frac{1}{4 \sqrt{\pi S}}\left(1+\frac{3 S}{\pi l^{2}}\right)
$$




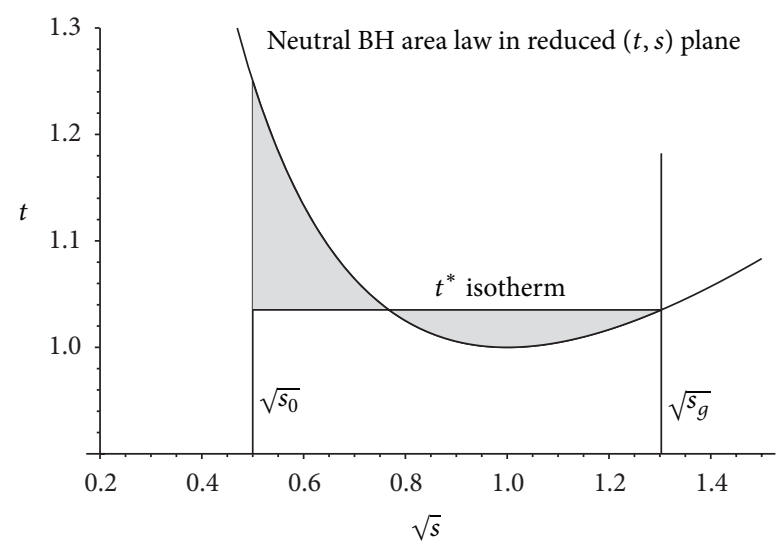

FIgURE 4: Plot of the temperature as function of the entropy. Equal are regions area shown in black. $s_{0}, s_{g}, t^{*}$ are given in (30), (31), and (33).

and plotted in Figure 4. The graph has a minimum at $S_{\min }=$ $\pi l^{2} / 3$ and $k_{B} T_{\min }=\sqrt{3} / 2 \pi l$. By rescaling (24) with respect to these extremal values, we obtain the reduced form

$$
\begin{gathered}
t=\frac{1}{2} \sqrt{s}+\frac{1}{2} \frac{1}{\sqrt{s}}, \\
s \equiv \frac{S}{S_{\min }}, \\
t \equiv \frac{T}{T_{\min }},
\end{gathered}
$$

which is valid for any value of the cosmological constant. The Maxwell area law follows from requirement that the Gibbs free energy is the same for coexisting BHs. One finds

$$
\begin{gathered}
G=M-T S, \\
\Delta G_{1,2}=-\int_{1}^{2} S d T=0, \\
T^{*} \Delta S_{1,2}=\int_{1}^{2} T d S,
\end{gathered}
$$

which leads to the definition of the equal area isotherm as follows:

$$
T^{*}=\frac{1}{2 \sqrt{\pi}\left(\sqrt{S_{1}}+\sqrt{S_{2}}\right)}\left[1+\frac{8 P}{3}\left(S_{1}+S_{2}+\sqrt{S_{1} S_{2}}\right)\right] .
$$

Reduced entropy solutions to the equal area law are obtained solving simultaneously the following system:

$$
\begin{gathered}
t=\frac{1}{2 \sqrt{s}}(s+1), \\
t^{*}=\frac{1}{\left(\sqrt{s_{0}}+\sqrt{s}\right)}\left[1+\frac{1}{3}\left(s_{0}+s+\sqrt{s_{0} s}\right)\right] .
\end{gathered}
$$

Introducing $\sqrt{s} \equiv x$, the above system leads to the following equation:

$$
2 x^{3}+x^{2}-7 x+3=0
$$

which has the following solutions:

$$
\begin{gathered}
\sqrt{s_{0}}=x_{1}=\frac{1}{2}, \\
\sqrt{s_{g}}=x_{2}=\frac{1}{2}(\sqrt{13}-1), \\
x_{3}=-\frac{1}{2}(\sqrt{13}+1), \\
t^{*}=\frac{1}{6}(2 \sqrt{13}-1),
\end{gathered}
$$

where $x_{3}$ is an unphysical solution leading to negative entropy, and $t^{*}$ is the Maxwell isotherm.

Maxwell construction has allowed us to eliminate negative specific heat part in the temperature graph. The motivation for removing this part of the temperature graph comes from the fact that it has no plausible physical explanation and does not allow the system to be in equilibrium with the heat bath. In the $(T, S)$ plane it is the analogue of the unstable (unphysical) part of the VdW graph in the $(P, V)$ plane. The flattening of the temperature graph through the isothermal cut modifies the Hawking-Page phase transition in the following way.

(i) Pure radiation phase survives beyond $T_{\min }=$ $\sqrt{3} / 2 \pi l$ up to the higher temperature, given by the isotherm $T^{*}$ :

$$
k_{B} T^{*}=\frac{\sqrt{3}}{12 \pi l}(2 \sqrt{13}-1) .
$$

(ii) For $T=T^{*}$, multiple, but stable, BHs form of different entropy. There are no more locally stable BHs that can decay into radiation as it occurs in Hawking-Page picture in the interval $T_{\min }<T<T_{\mathrm{HP}}$.

(iii) For $T>T^{*}$, there exists single, stable $\mathrm{BH}$ with positive heat capacity.

One may be tempted to ask what kind of statistical ensemble describes the $\mathrm{BH}$ thermodynamics.

Hawking has argued that the suitable description is in terms of canonical ensemble with the generating function to be defined in terms of Helmholtz free energy $F=$ $M-T S$. This seems to be a suitable picture as long as the cosmological constant is held fixed. On the other hand, when one decides to work in an extended phase space $[7,10]$, the same function is interpreted as the Gibbs free energy and, thus, the suitable description should be in terms of grand canonical ensemble, since the $P V$ term, a.k.a. the q-potential, defines the generating function for this ensemble. Since in this paper we put in evidence the $P V$ term, and allow $P$ to vary in the $(P, V)$ plane, we are actually dealing with the grand canonical ensemble. An additional motivation is that the Maxwell area construction is based on the Gibbs free energy characterizing the grand canonical ensemble.

In our view, a statistical description can be only formal, until a microscopic structure of the $\mathrm{BH}$ is properly 
understood in terms of a reliable theory of quantum gravity. Nevertheless, working with different ensembles is just a matter of convenience leading to the same equation of state (19).

\section{Conclusions}

In this paper, we have presented a new scenario for Schwarzschild $\mathrm{BH}$ nucleation in the AdS vacuum. This kind of BHs has a well-known, but largely ignored, problem concerning the negative heat for $r_{+}<r_{\min }=l / \sqrt{3}$. Similar negative specific heat regions exist also for other types of BHs. So far, no plausible physical explanation for negative heat capacity has been given. The problem of negative heat capacity is related to the fact that such BHs cannot be in thermal equilibrium with the background; thus, they cannot slowly evolve through a series of successive equilibrium states at different temperature. To avoid these problems, we construct an isothermal cut in the temperature graph which eliminates negative heat capacity region, following the Maxwell equal area prescription in the $(T, S)$ plane.

We also studied the effect of the Maxwell construction on the Hawking-Page transition picture. It turns out that there are significant modifications: BHs start nucleating at a temperature $T^{*}$ higher than the temperature $T_{\min }=\sqrt{3} / 2 \pi l$ as in the original Hawking-Page scenario. Above $T^{*}$, there are only single stable BHs. Therefore, having removed the negative specific heat region, unstable $\mathrm{BH}$ cannot be formed. Along the isotherm $T=T^{*}$, BHs of different radii have the same negative free energy and from a statistical point of view are equally probable among themselves, but more stable than the pure radiation. Degeneracy between radiation and $\mathrm{BHs}$ has been removed since $T^{*}>T_{\mathrm{HP}}$.

We have also discussed the corresponding equation of state $P=P(V)$, where the pressure is related to the cosmological constant and $V$ corresponds to the geometric volume of the $\mathrm{BH}$. In the literature, different authors make different choices for the volume to be used in the equation of state, and it seems that there is no unique choice that everybody agrees upon. We have given our contribution to this controversy based on the following argument: if a gas equation is to truly describe a $\mathrm{BH}$; it has to reproduce its basic properties and, in particular, area/entropy relation should follow from thermodynamical arguments. We have shown by an explicit calculation that the geometrical volume $V=$ $4 \pi r_{H}^{3} / 3$ is needed to obtain the correct entropy, at least in the model described in this paper.

\section{References}

[1] D. Kastor, S. Ray, and J. Traschen, "Enthalpy and the mechanics of AdS black holes," Classical and Quantum Gravity, vol. 26, no. 19, Article ID 195011, 2009.

[2] A. Chamblin, R. Emparan, C. V. Johnson, and R. C. Myers, "Charged AdS black holes and catastrophic holography," Physical Review D, vol. 60, no. 6, Article ID 064018, 17 pages, 1999.

[3] A. Chamblin, R. Emparan, C. V. Johnson, and R. C. Myers, "Holography, thermodynamics, and fluctuations of charged
AdS black holes," Physical Review D, vol. 60, no. 10, Article ID 104026, 20 pages, 1999.

[4] Y. D. Tsai, X. N. Wu, and Y. Yang, "Phase structure of the KerrAdS black hole," Physical Review D, vol. 85, no. 4, Article ID 044005, 9 pages, 2012.

[5] P. Nicolini and G. Torrieri, "The Hawking-Page crossover in noncommutative anti-deSitter space," Journal of High Energy Physics, vol. 2011, no. 8, article 97, 2011.

[6] B. P. Dolan, "The cosmological constant and black-hole thermodynamic potentials," Classical and Quantum Gravity, vol. 28, no. 12, Article ID 125020, 2011.

[7] B. P. Dolan, "Pressure and volume in the first law of black hole thermodynamics," Classical and Quantum Gravity, vol. 28, Article ID 235017, 2011.

[8] B. P. Dolan, "Compressibility of rotating black holes," Physical Review D, vol. 84, no. 12, Article ID 127503, 3 pages, 2011.

[9] B. P. Dolan, "Where is the PdV term in the first law of black hole thermodynamics?" in Open Questions in Cosmology, InTech, 2012.

[10] M. M. Caldarelli, G. Cognola, and D. Klemm, "Thermodynamics of Kerr-Newman-AdS black holes and conformal field theories," Classical and Quantum Gravity, vol. 17, no. 2, pp. 399$420,2000$.

[11] M. Cvetic, G. W. Gibbons, D. Kubiznak, and C. N. Pope, "Black hole enthalpy and an entropy inequality for the thermodynamic volume," Physical Review D, vol. 84, no. 2, Article ID 024037, 17 pages, 2011.

[12] H. Lu, Y. Pang, C. N. Pope, and J. F. Vazquez-Poritz, "AdS and Lifshitz black holes in conformal and Einstein-Weyl gravities," Physical Review D, vol. 86, no. 4, Article ID 044011, 22 pages, 2012.

[13] R. Banerjee, S. Ghosh, and D. Roychowdhury, "New type of phase transition in Reissner Nordström-AdS black hole and its thermodynamic geometry," Physics Letters B, vol. 696, no. 1-2, pp. 156-162, 2011.

[14] R. Banerjee and D. Roychowdhury, "Thermodynamics of phase transition in higher dimensional AdS black holes," Journal of High Energy Physics, vol. 2011, no. 11, article 4, 2011.

[15] R. Banerjee, S. K. Modak, and D. Roychowdhury, "A unified picture of phase transition: from liquid-vapour systems to AdS black holes," Journal of High Energy Physics, vol. 2012, no. 10, article 125, 2012.

[16] A. Smailagic and E. Spallucci, "Thermodynamical phases of a regular SAdS BH," International Journal of Modern Physics D, vol. 22, no. 3, Article ID 1350010, 12 pages, 2013.

[17] E. Spallucci and A. Smailagic, "Maxwell's equal-area law for charged Anti-de Sitter black holes," Physics Letters B, vol. 723, no. 4-5, pp. 436-441, 2013.

[18] S. W. Hawking and D. N. Page, "Thermodynamics of black holes in anti-de Sitter space," Communications in Mathematical Physics, vol. 87, no. 4, pp. 577-588, 1983.

[19] E. Witten, "Anti-de Sitter space, thermal phase transition, and confinement in gauge theories," Advances in Theoretical and Mathematical Physics, vol. 2, pp. 505-532, 1998.

[20] E. Witten, "Anti-de Sitter space and holography," Advances in Theoretical and Mathematical Physics, vol. 2, pp. 253-291, 1998.

[21] R. C. Myers and S. E. Vazquez, "Quark soup al dente: applied superstring theory," Classical and Quantum Gravity, vol. 25, no. 11, Article ID 114008, 2008. 
[22] D. Kubiznak and R. B. Mann, " $P-V$ criticality of charged AdS black holes," Journal of High Energy Physics, vol. 2012, no. 7, article 33, 2012.

[23] S. Gunasekaran, D. Kubiznak, and R. B. Mann, "Extended phase space thermodynamics for charged and rotating black holes and Born-Infeld vacuum polarization," Journal of High Energy Physics, vol. 2012, no. 11, article 110, 2012. 

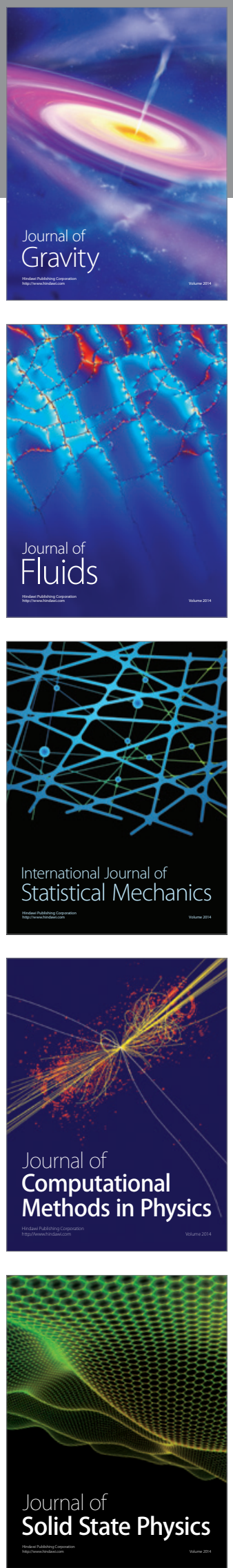

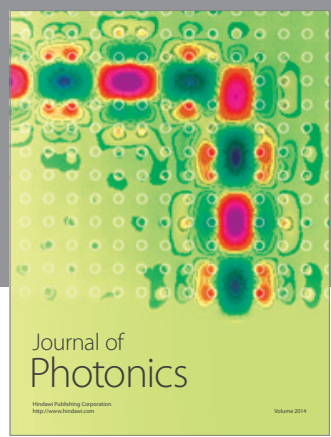

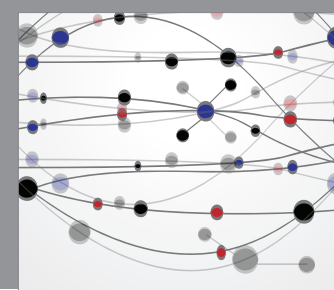

The Scientific World Journal

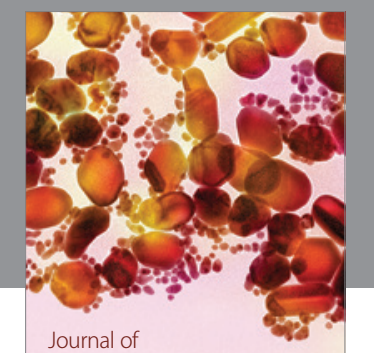

Soft Matter
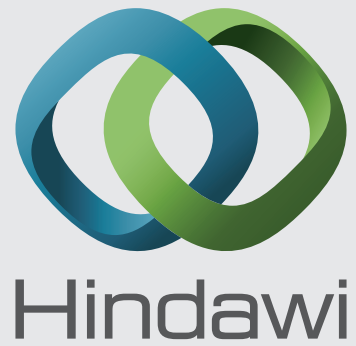

Submit your manuscripts at

http://www.hindawi.com
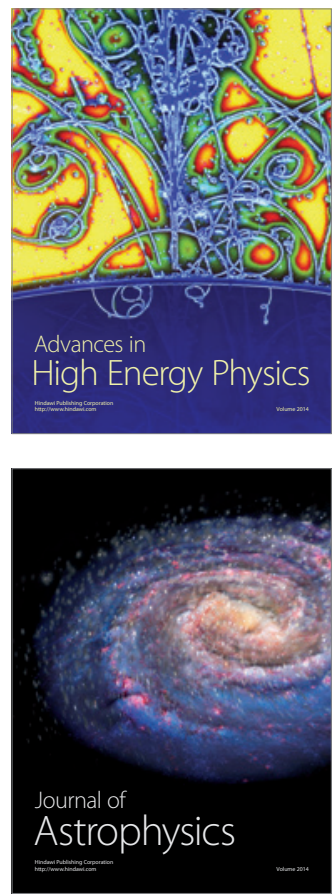
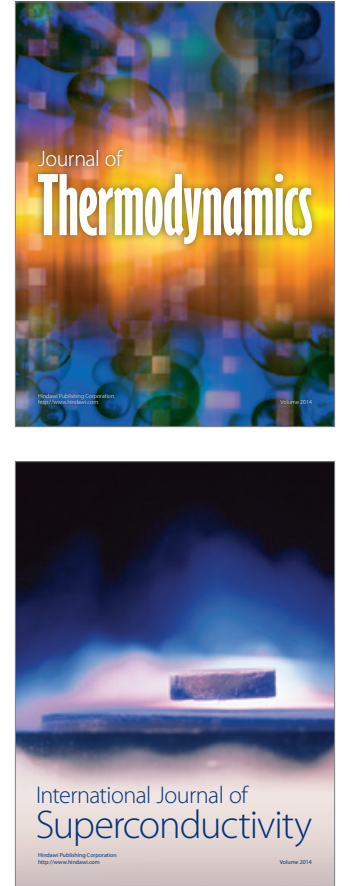
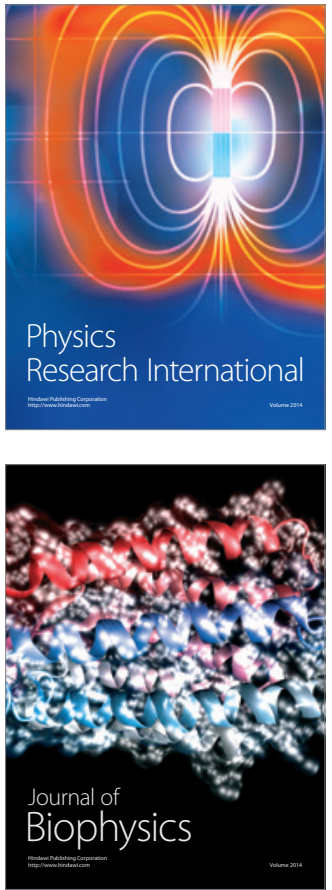
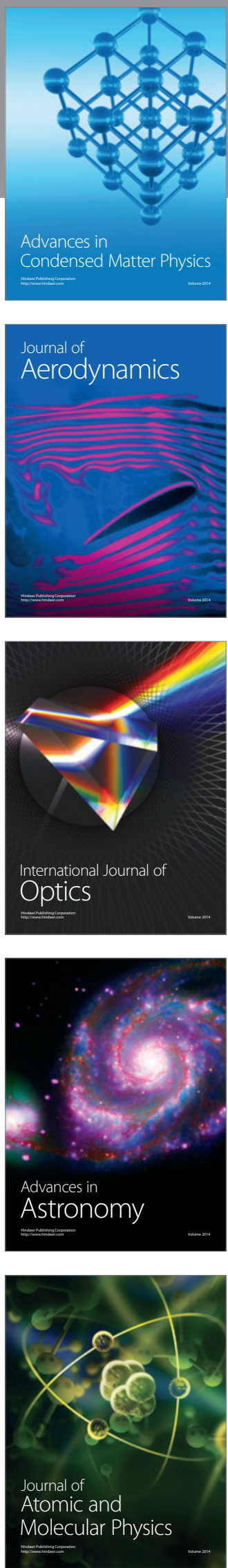